\title{
Expression of VEGF and Nestin in Chronic Restraint-Induced Stress in the Livers of Male Albino Rats.
}

Hassan Reda Hassan Elsayed

lecturer of Anatomy and Embryology,

faculty of Medicine Mansoura university,

\begin{abstract}
Background and Aim of work:

Stress and its related comorbid diseases are responsible for many liver pathologies. Nestin, a protein detected in a wide range of progenitor cells, was implicated in hepatic remodeling. Moreover, vascular endothelial growth factor (VEGF) is involved in vessel repair. The aim of this study was to evaluate the effect of chronic restrain stress (CRS) on expression of hepatic VEGF and Nestin.

Materials and methods:

Twelve male rats were divided into two groups; negative control and CRS. Animals of CRS were restrained individually in ventilated glass tube restrainers daily for 6 hours/day for 3 weeks. The liver samples were stained by hematoxylin and eosin, Masson trichrome and immunohistochemical staining for Nestin and VEGF with morphometric analysis.

Results:

Exposure to chronic restraint stress for 3 weeks caused hepatic inflammation, deposition of collagen. Immunohistochemical staining showed a coinciding significantly higher positive reaction for VEGF and Nestin in the CRS group.

Conclusion:

Vascular endothelial growth factor and Nestin immunohistochemical staining were both increased in chronic restraint-induced hepatic stress coinciding with the induced increase in hepatic inflammation, deposition of collagen. Nestin \& VEGF expressions were strongly correlated. This may be due to that the Nestin is expressed in the new endothelial cells and is responsible for their migration after the stress induced hepatic injury. However, the regulatory relationship between Nestin and VEGF is still not clear.
\end{abstract}

\section{Introduction}

Stress can be considered as a disrupted homeostasis triggered by a physiological, environmental or psychological factors. The adaptation to these factors includes the stimulation of the hypothalamus-pituitary-suprarenal hormonal axis, with concomitant release of catecholamines, glucocorticoids (GCs). Stress is associated with circulatory disturbance, hypoxia and oxidative stress in body organs including liver (Jafari et al., 2014).

Nestin belongs to class $\mathrm{VI}$ of the intermediate filament protein, related to vimentin, desmin and GFAP; class III intermediate filament proteins (Toi et al., 2018). Nestin is not only expressed in neural precursor cells, but is also expressed in immature neuroglia, reactive astrocytes, neuroectodermal cancers, melanomas, immature skeletal and cardiac muscle cells; all of these cells have a common neural crest origin (Shi et al., 2016). However, Nestin is strongly expressed in endothelial cells in response to pathological neovascularization, as usually found in acute pancreatitis, glycuresis, and nephropathy (Liang et al., 2015). 
The endothelial cells' migration is an important step in neovascularization. VEGF is a molecule signaling for triggering migration of $\mathrm{EC}$ in neovascularization (Martinez et al., 2015). Although both Nestin and VEGF play significant roles in neovascularization, the regulatory relationship between them is still not clear (Liang et al., 2015).

The aim of the present work was to evaluate the Nestin \& VEGF immunoreactivity in the male albino rat liver of a chronic restraint stress model and the correlation between Nestin and VEGF.

\section{Material and Methods}

\section{Animals}

The study used 12 male adult albino rats weighing 200-250 gm and ages around 10 weeks. The rats were kept in standard conditions in the laboratory with normal cycle of day / night for 2 weeks before initiating the study. The rats were fed a basal diet and tap water ad libitum. The animal care and experiments protocol described in this study met the terms of "Committee of Research Ethics" in the Faculty of Medicine, Mansoura University, Egypt, meeting the guidelines of National Institute of Health $(\mathrm{NIH})$ and Institutional Research Board (IRB) for animal use.

Animals were divided into the 2 groups (6 rats in each group):

1. Negative control group: animals were kept untreated with no stress.

2. Chronic restraint Stress (CRS) group: animals were restrained individually in ventilated glass tube restrainers for 3 weeks about 6 hours/day (9 AM - 3 PM) with no access to water and food throughout the stress hours. After the stress protocol, animals will be back to their cages with water and food ad libitum during the other 18 hours (Srikumar et al., 2007). Rats were sacrificed by performing decapitation at the end of stress exposure period.

\section{Histolological examination}

Liver samples from the 2 groups were obtained and exposed to fixation in $10 \%$ buffered formaldehyde and paraffin-embedded. Liver samples have been cut in 5 um thick sections of tissues and then stained in hematoxylin and eosin, (H\&E); and Masson trichrome (Bancroft \& Gamble, 2008) and then examined by light microscopy.

Immunostaining analyses were performed on formalin-fixed, paraffin-embedded samples. Liver samples were cut serially at $5 \mu \mathrm{m}$ thick sections. Liver sections were deparaffinized by xylene then rehydrated in graded ethyl alcohol. Then the liver sections were kept in 3\% $\mathrm{H} 2 \mathrm{O} 2$ for 5 - 10 minutes at room temperature to eliminate intrinsic peroxidase activity. Then they were put in $10 \mathrm{mM}$ citrate buffer at $\mathrm{pH} 6$ for 10 minutes at $95 \mathrm{C}-100 \mathrm{C}$ and allowed to cool at room temperature for 60 minutes for antigen retrieval. Then the slides were incubated with the primary antibody (Liu et al., 2017).

A-Immunohistochemistry for nestin protein expression in the liver was performed by antinestin antibody. The primary antibody used was a rabbit polyclonal antibody (N5413, Sigma Chemicals Co., St. Louis, MO, USA) at 1:100 dilution. It was raised against recombinant nestin of human origin and specific for nestin of human, rat and mice (Klein et al., 2014).

B- Immunohistochemistry for VEGF expression in the liver tissue were performed by a rabbit polyclonal Anti-VEGF antibody (ab16883, Abcam, Cambridge, UK) at a 1:200 dilutions. It is used as a specific marker of angiogenesis (Cheng et al., 2011).

Then the sections were left over night at room temperature (RT). After 3 rinses with phosphatebuffered saline, the secondary antibody was added for 30 minutes at RT then the horseradish peroxidase streptavidin complex was applied for 60 
minutes at RT, then visualized by diaminobenzidine chromogen solution and counterstained with hematoxylin for 5 minutes. The sections were washed, dehydrated, then mounted, and examined. Negative controls were assessed by replacing the primary antibody by PBS (Ramos-Vara and Miller, 2014). All sections were examined using light microscope.

\section{Morphometric study}

The mean number of VEGF and Nestin immunolabeled cells was measured in 10 nonoverlapping fields of liver sections in the rats of all groups.

\section{Statistical analysis of data}

Data analysis was expressed as mean \pm standard deviation. Independent-samples t-test was done. Data were considered statistically significant when $p \leq 0.05$. We used SPSS software for all statistical analyses.

\section{Results :}

H\&E light microscopic results

Control group showed normal liver histology represented by the cords of polyhedral hepatocytes in a radial form with the central vein (CV) in the center. Cytoplasm of hepatocytes was acidophilic and nuclei were vesicular and central. Blood sinusoids separate the hepatic cords. Portal tracts were normal (Figure 1a, b). Stress group showed multiple inflammatory cells in the sinusoidal lumen around the CV and disrupted EC with inflam- matory cells escape through hepatic cords. Moreover, there were necrotic foci and occasional hepatocyte degeneration. Some hepatic cells showed acidophilic cytoplasm with pyknotic small nuclei. Neutrophils infiltrated the Portal tracts (Figure 1c, d).

\section{Masson trichrome sections}

Liver of the control group showed fine fibers of collagen surrounding the portal tracts and the central veins (Fig. 2A), while the livers of the CRS group (fig2B): showed dense fibers of collagen surrounding the portal tracts and the central veins.

\section{Immunohistochemical study of Nestin}

Liver sections immunolabeled for Nestin showed mild expression in the control group (fig3a, b). While there was a high nestin expression in the cytoplasm of many hepatocytes of the 3 weeks' stress group (Figure 3c, d).

\section{Immunohistochemical study of VEGF}

Liver sections immunostained with VEGF in the control group showed mild expression (fig4A, B). While, there was a high cytoplasmic VEGF expression in endothelial cells and hepatocytes in 3 weeks CRS group (Fig 4C, D)

\section{Morphometric results}

Our data showed that there was significant increase in the mean number of Nestin \& VEGF immunopositive cells in 3 weeks CRS group compared to the control group (Table 1). 
Table 1. number of Nestin \& VEGF immunolabeled cells

\begin{tabular}{lll}
\hline & Control & 3 weeks CRS \\
\hline Mean number of Nestin immunolabeled cells & $34.25 \pm 6.55$ & $\mathbf{1 8 0 . 7 2 \pm 4 3 . 0 3 ^ { * }}$ \\
\hline Mean number of VEGF immunolabeled cells & $19.3 \pm 7.33$ & $66.98 \pm 11.76^{*}$ \\
\hline
\end{tabular}

Data are presented as mean \pm standard deviation.

${ }^{*}=p \leq 0.05$ means statistically significant in comparison to the control group
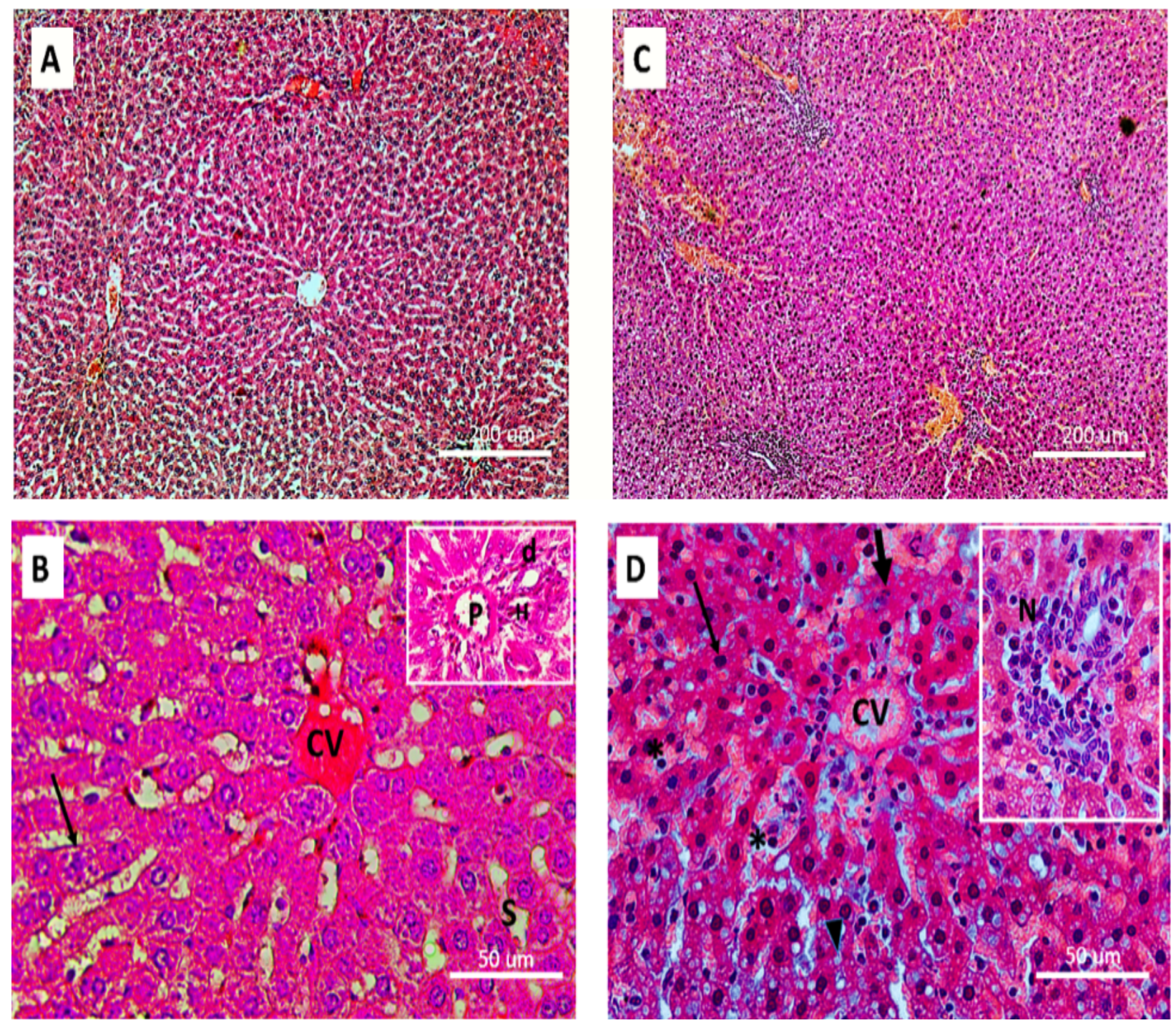

Figure (1): Photomicrograph of the Control group (Fig1a, b) showing normal liver histology represented by the cords of polyhedral hepatocytes (arrow) in a radial form with the central vein $(\mathrm{CV})$ in the center. Cytoplasm of hepatocytes is acidophilic and nuclei are vesicular and central. Blood sinusoids (S) separate the hepatic cords. Portal tracts are normal (as seen in the inset; Hepatic artery (H) Portal vein (P), Bile duct (D). Stress group (Fig1c, d) shows multiple inflammatory cells in the sinusoidal lumen $(*)$ around the CV and disrupted EC with of inflammatory cells escape through hepatic cords. Moreover, there are necrotic foci and occasional hepatocyte degeneration (thick arrow). Some hepatic cells show acidophilic cytoplasm with pyknotic small nuclei (thin arrow). Vacuolated cytoplasm by microvesicular steatosis (arrow heads). Neutrophils (N) infiltrated the Portal tracts (in the inset) (Figure 1d) (hematoxylin and eosin, A, C*100, B,D *400) 

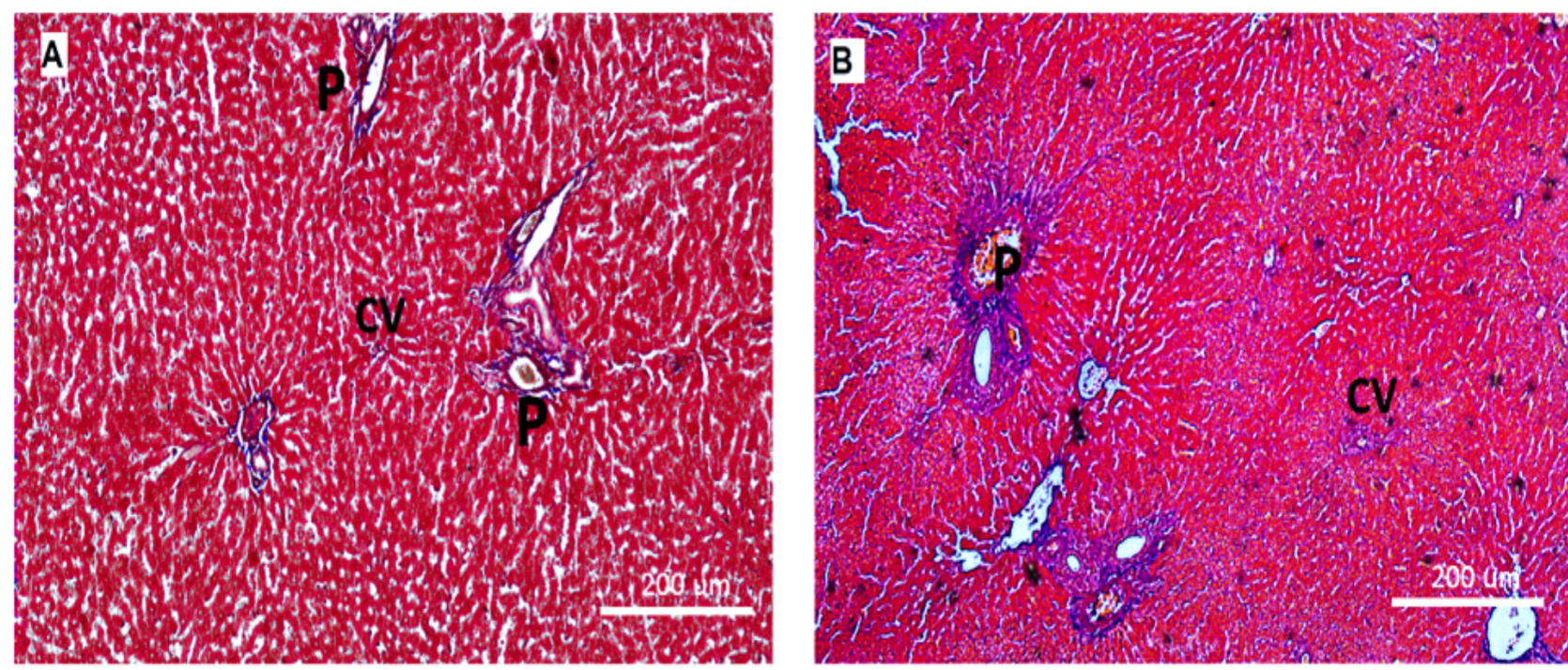

Figure (2): Photomicrograph of Liver of the control group shows fine fibers of collagen surrounding the portal tracts (P) and the central veins (CV) (Fig. 2a), while the livers of the CRS group (fig2B): show dense fibers of collagen surrounding the portal tracts $(\mathrm{P})$ and the central veins. Masson's trichrome, X 100
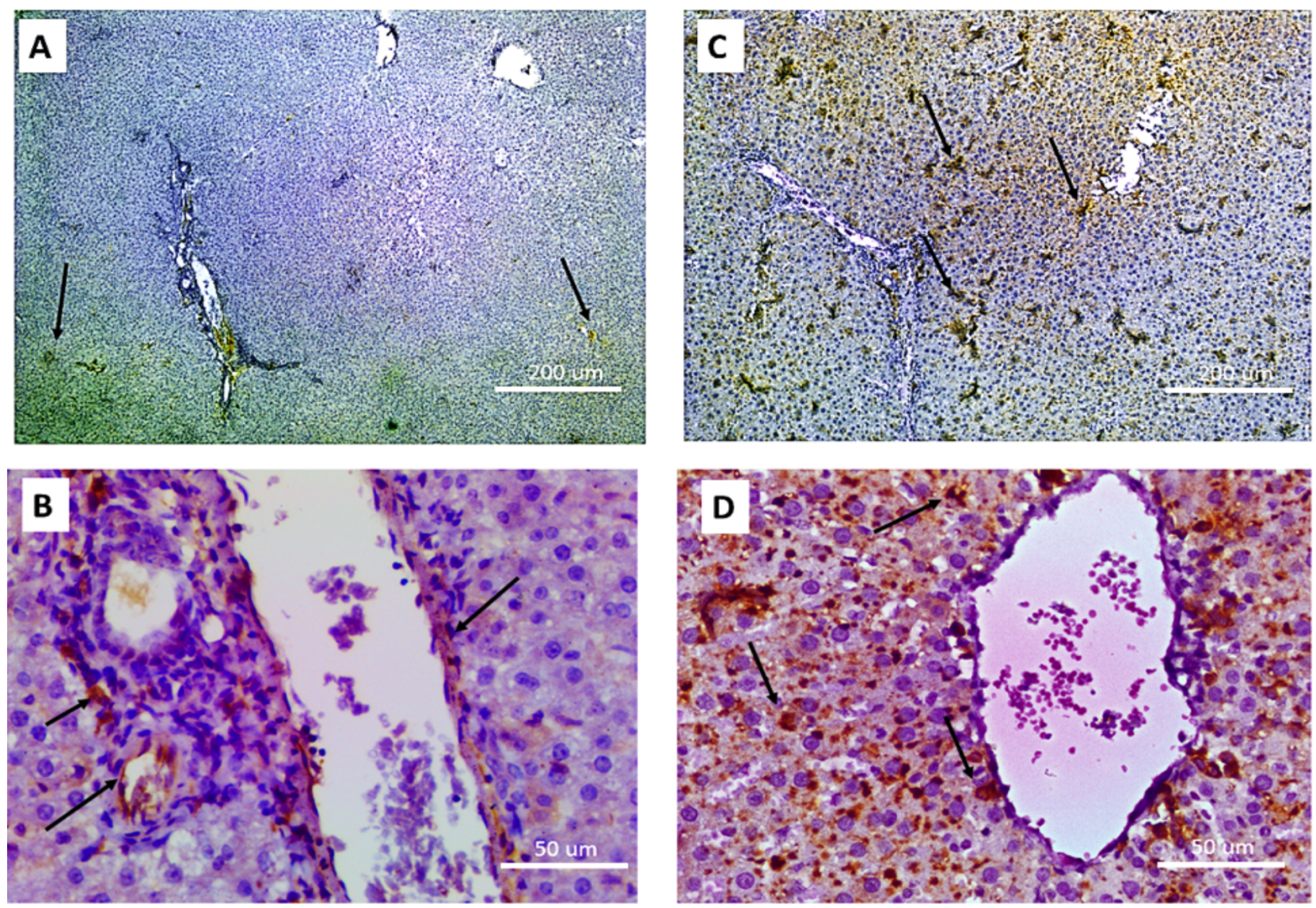

Figure 3: Photomicrographs immunostained for Nestin: control group (fig3A, B) and 3weeks CRS group (fig3C, D) showing: higher cytoplasmic expression (arrows) in the stress group in many hepatocytes and endothelial cells. (immunohistochemistry for nestin, A, C*100, B, D *400) 

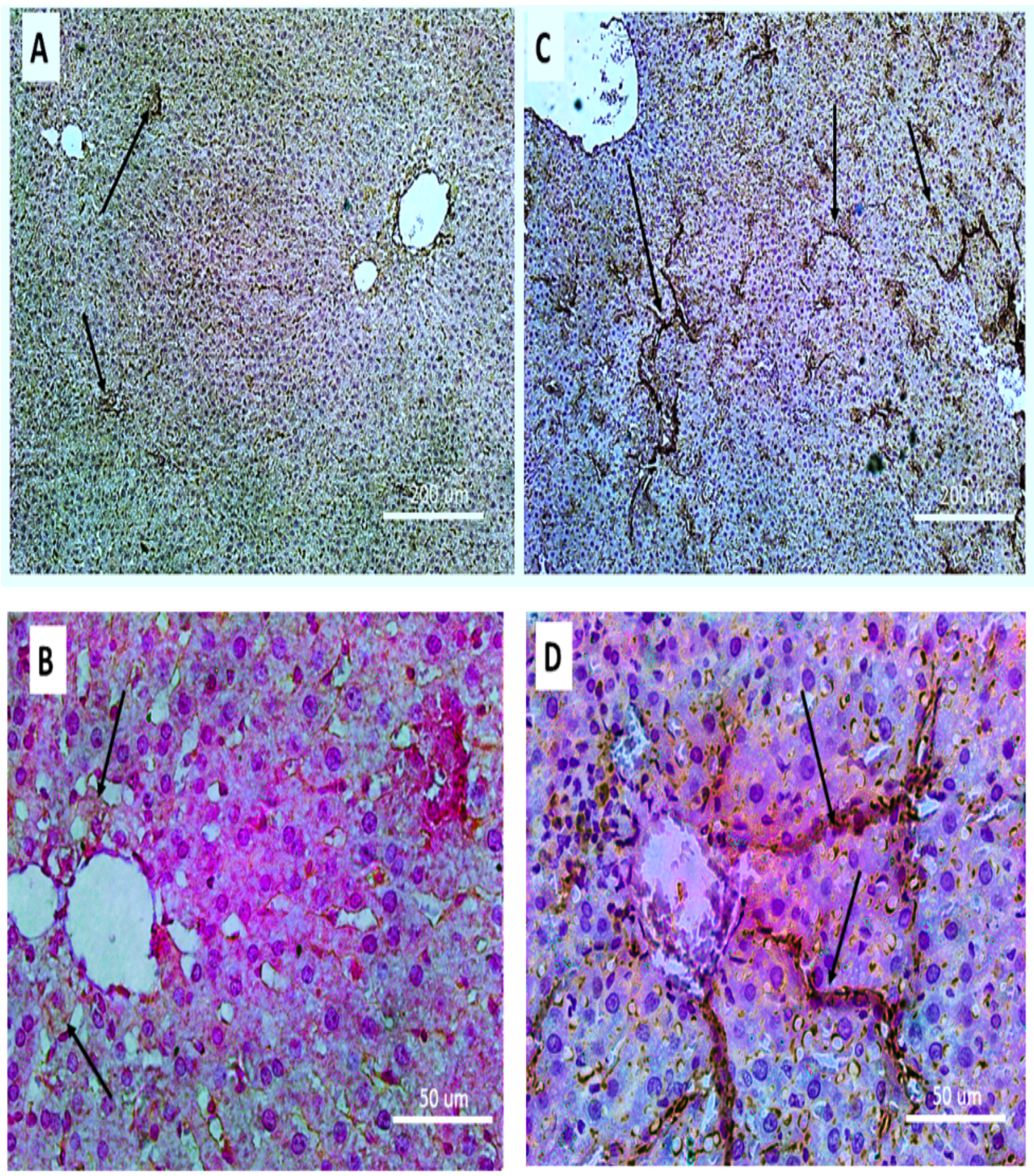

Figure (4): Photomicrographs immunolabeled for VEGF: control group (Fig4A, B) and 3 weeks CRS group (fig4C, D) showing high expression in endothelial cells and hepatocytes (arrows). (Immunohistochemistry; A, C*100, B, D *400) 


\section{Discussion}

Chronic restraint stress-induced liver injury as seen in our results can be considered as a disrupted homeostasis triggered by a physiological, environmental or psychological factors. Stress and its related comorbid diseases were found to be responsible for many liver pathologies. The finding of liver injury, inflammation and fibrosis in CRS group can be explained by the fact the adaptation response to stress is associated with circulatory disturbance, hypoxia in body organs (Jafari, et al., 2014).

Stress involves liver through modulation of GCs level in serum which is thought to enhance the toxic effect of oxygen free radical producers. Moreover, activated lipid peroxidation after Stress can cause distortion to cellular ultrastructure with hepatocytes necrosis (Djordjevic et al., 2010).

The finding of increased Nestin expression in the CRS group can be explained by the fact that Nestin is one of Intermediate filaments (IFs) which are cytoskeletal proteins, critical for regulation of cell migration and maintenance of cell shape (Toi et al., 2018) and that the Nestin is highly expressed in endothelial cells in cases of pathological neovascularization and that Nestin could also induce proliferation and migration of ECs by acting as a survival factor (Liang et al., 2015). Additionally, Nestin expression was found in immature RECA-1 +ve endothelial cells (Golbar et al., 2014). Furthermore, Nestin is expressed in HSCs responsible for fibrogenesis after liver injury (Toi et al., 2018). Moreover, Nestin is also expressed in hepatic oval progenitor cells during regeneration (Koenig et al., 2006). Nestin was also expressed in $\mathrm{CK} 19$ +ve regenerating cholangiocytes (Golbar et al., 2014).

Migration of vascular ECs requires the cytoskeleton and its binding proteins. Both cytoskele- ton arrangement and configurational changes occur in endothelial cells, triggered by chemokines, matrix proteins and growth factors that cause cell polarization (Toi et al., 2018).

The finding of increased VEGF expression in CRs group can be explained by the fact that stress-induced hepatic injury triggers the migration of ECs and angiogenesis and that VEGF is usually considered as a triggering molecule for the molecule for EC migration. VEGF is involved in pathological neovascularization but could also play a role in physiological revascularization, regeneration, platelet secretion and haemostasis (Martinez et al., 2015).

The finding of concomitant increased expression of both Nestin \& VEGF can be explained by the fact that VEGF can affect the cytoskeleton to stimulate the migration of vascular endothelial cells. Moreover, the colocalization of CD31 (endothelial marker for new blood vessels) and Nestin during neovascularization in injured rat corneas confirmed the role of nestin in angiogenesis. Furthermore, the exposure of endothelial cells from human umbilical vein to hypoxia could trigger Nestin expression. Additionally, silencing of Nestin through siRNA suppressed VEGF-induced neovascularization, as tube formation and the proliferation and migration of the endothelial cells from human umbilical vein. Also, F-actin filaments were found to be successfully arranged into microfilaments when cells were treated with VEGF, proposing a network organization performed by $\mathrm{F}$ actin that was in contrast with the loose and uniform actin filament network found in siRNA-Nestin cells. Moreover, Nestin-mediated remodeling of cytoskeleton in ECs was found to be performed by formation of filopodia alongside the cellular margin, triggering both localization of filopodia and polarization of cells and finally triggering ECs migration induced by VEGF, thought to be related 
to ERK pathway activation. Lastly, although both VEGF and Nestin play important roles in angiogenesis, the regulatory relationship between them still need more research (Liang et al., 2015).

\section{References}

Bancroft, J. D., \& Gamble, M. (2008): Theory and practice of histological techniques: Elsevier Health Sciences.

Cheng, S. J., Lee, J. J., Kok, S. H., Chou, C. H., Chang, H. H., Yang, H., ... Kuo, M. Y. (2011). Expression of vascular endothelial growth factor is significantly associated with progression and prognosis of oral squamous cell carcinomas in Taiwan. J Formos Med Assoc, 110(1), 50-57. doi:10.1016/S0929-6646(11)60008-9.

Djordjevic, J., Djordjevic, A., Adzic, M., Niciforovic, A., \& Radojcic, M. B. (2010). Chronic stress differentially affects antioxidant enzymes and modifies the acute stress response in liver of Wistar rats. Physiol Res, 59(5), 729-736.

Golbar, H. M., Izawa, T., Wijesundera, K. K., Tennakoon, A. H., Katou-Ichikawa, C., Tanaka, M., . . Yamate, J. (2014). Expression of nestin in remodelling of alpha-naphthylisothiocyanateinduced acute bile duct injury in rats. J Comp Pathol, 151(2-3), 255-263. doi:10.1016/ j.jcpa.2014.05.003.

Jafari, M., Salehi, M., Zardooz, H., \& Rostamkhani, F. (2014). Response of liver antioxidant defense system to acute and chronic physical and psychological stresses in male rats. EXCLI J, 13, 161-171.

Johnson, K. E., \& Wilgus, T. A. (2014). Vascular Endothelial Growth Factor and Angiogenesis in the Regulation of Cutaneous Wound Repair. Adv Wound Care (New Rochelle), 3(10), 647661. doi:10.1089/wound.2013.0517.
Klein, D., Meissner, N., Kleff, V., Jastrow, H., Yamaguchi, M., Ergun, S., \& Jendrossek, V. (2014). Nestin(+) tissue-resident multipotent stem cells contribute to tumor progression by differentiating into pericytes and smooth muscle cells resulting in blood vessel remodeling. Front Oncol, 4, 169. doi:10.3389/ fonc.2014.00169.

Koenig, S., Probst, I., Becker, H., \& Krause, P. (2006). Zonal hierarchy of differentiation markers and nestin expression during oval cell mediated rat liver regeneration. Histochem Cell Biol, 126(6), 723-734. doi:10.1007/s00418006-0204-3.

Liang, Z. W., Wang, Z., Chen, H., Li, C., Zhou, T., Yang, Z., . . . Cai, W. (2015). Nestin-mediated cytoskeletal remodeling in endothelial cells: novel mechanistic insight into VEGF-induced cell migration in angiogenesis. Am J Physiol Cell Physiol, 308(5), C349-358. doi:10.1152/ ajpcell.00121.2014.

Liu, F., Zhang, Y., Lu, M., Wang, C., Li, Q., Gao, Y., . . Meng, X. (2017). Nestin servers as a promising prognostic biomarker in non-small cell lung cancer. Am J Transl Res, 9(3), 13921401.

Martinez, C. E., Smith, P. C., \& Palma Alvarado, V. A. (2015). The influence of platelet-derived products on angiogenesis and tissue repair: a concise update. Front Physiol, 6, 290. doi:10.3389/fphys.2015.00290.

Ramos-Vara, J. A., \& Miller, M. A. (2014). When tissue antigens and antibodies get along: revisiting the technical aspects of immunohistochemistry--the red, brown, and blue technique. Vet Pathol, 51(1), 42-87. doi:10.1177/ 0300985813505879.

Shi, A. M., Tao, Z. Q., Wang, X., \& Wang, Y. Q. 
(2016). Intermediate filament Nestin and the cell motility in cancer - a review. Eur Rev Med Pharmacol Sci, 20(17), 3514.

Srikumar, B. N., Raju, T. R., \& Shankaranarayana Rao, B. S. (2007). Contrasting effects of bromocriptine on learning of a partially baited radial arm maze task in the presence and absence of restraint stress. Psychopharmacology (Berl),
193(3), 363-374. doi:10.1007/s00213-0070801-4.

Toi, M., Hayashi, Y., \& Murakami, I. (2018). Hepatic stellate cells derived from the nestin-positive cells in septum transversum during rat liver development. Med Mol Morphol, 51(4), 199-207. doi:10. 1007/s00795-0180183-1. 